\title{
Internacionalización y localización del software en el ámbito mundial
}

\section{Internationalization and localization of software worldwide}

Gilberto Fernando Castro Aguilar

Universidad de Guayaquil, Ecuador

Universidad Católica de Santiago de Guayaquil, Ecuador

Yulia Fustiel Álvarez

Universidad de las Ciencias Informáticas, Cuba.

Francisco Palacios Ortiz

Iván Cabrera Salazar

Raúl Omar Cárdenas Marín

Universidad de Guayaquil, Ecuador

Autor para la correspondencia: gilberto.castroa@ug.edu.ec,yfustiel@uci.cu, francisco.palacioso@ug.edu.ec, ivan.cabreras@ug.edu.ec, raul.cardenasm@ug.edu.ec

Fecha de recepción: 01 de Febrero de 2017 - Fecha de aceptación: 10 de Agosto de 2017

Resumen: La expansión del mercado a nivel internacional es una de las características más importantes del desarrollo económico de las instituciones en todo el mundo, lo que conlleva a lograr la introducción de los productos de software en mercados internacionales destinos. En este sentido las instituciones deben empeñarse de manera ardua en la adaptación de sus procesos, procedimientos y los propios productos. Las instituciones que estratégicamente deciden internacionalizarse tienen el reto de lograr que sus productos o servicios tengan una adecuada transformación lingüística, cultural y legal de forma que pueda ser adaptada a diferentes países. El desarrollo de productos o servicios internacionalizados es una tarea compleja y costosa debido a los altos costos de traducción de contenidos, el aumento de horas de trabajo en el diseño y la codificación, la necesidad de herramientas y habilidades adicionales, y los efectos sobre la gestión y la arquitectura del proyecto. La pronta detección de los requisitos de internacionalización por parte del arquitecto y líder de proyecto influye en la facilidad de implementación y en la detección y corrección de errores. El propósito de este trabajo es reflejar todos aquellos aspectos relacionados con los procesos de internacionalización y localización del software, a partir de un estudio actual acerca de estos temas que son muy necesarios para el desarrollo de productos y la prestación de servicios globalizados. Además se presentan directrices que sirven de apoyo a los equipos de proyectos que tradicionalmente han desarrollados productos de software a la medida.

Palabras Clave: globalización del software; internacionalización del software; i18n; localización del software; $110 \mathrm{n}$

Abstract: Market expansion is one of the most important characteristics of institutions economic development all over the world, it makes them to pursue introducing their software products in different international markets. Thus institutions must definitely focus on adapting their processes, procedures, and their own products. When internationalization becomes a strategic decision there is a challenge to achieve a proper linguistic, cultural, and legal transformation of products and services in order to be adapted to different countries. The development of internationalized 
products or services is a complex and costly task due to the high costs of content translation, increased working hours in design and coding, the need for additional tools and skills, and the effects on management and architecture of the project. The early detection of internationalization requirements by the architect and project leader influences the ease of implementation and the detection and correction of errors. The aim of this paper is to refer to all those aspects related to software internationalization and localization processes, based on a current study about these necessary topics for products development and globalized services presentation. In addition, guidelines are presented to support project teams that have traditionally developed custom software products.

Key words: software globalization; software internationalization; i18n; software localization; 110 n

\section{Introducción}

La Universidad Latinoamericana y el Caribe, en su camino de transformación ha ido tomando protagonismos trascendentes que se han reflejado en aportes importantes en la ciencia, en particular la universidad ecuatoriana, alineada a una exigencia mundial, ha adoptado modelos y mejores prácticas de aquellas que han tenido protagonismos importantes. Son los casos de la Universidad de Guayaquil (UG) y la Universidad Católica de Santiago de Guayaquil (UCSG), que están entre las universidades más importantes del Ecuador, y como proceso de crecimiento forman a profesionales en las áreas de tecnologías informáticas, con altos niveles de exigencia y compromiso, como versan sus misiones, visiones y objetivos. En este mismo principio de crecimiento y con un camino ya recorrido, la Universidad de las Ciencias Informáticas (UCI) de la Habana - Cuba, es una de las principales instituciones dedicadas al desarrollo de software y tiene como parte de su misión formar profesionales comprometidos con su patria y altamente calificados en la rama de la informática, capaces de producir aplicaciones y servicios informáticos de alto valor agregado a partir de la vinculación estudio-trabajo como modelo de formación.

Los profesores y especialistas no solo están llamados a formar parte de la principal fuerza que colabore con la estrategia de informatización de estas sociedades sino que además tienen el reto de llevar sus productos de software y servicios informáticos a mercados internacionales, de manera que estos puedan competir en todo el mundo.

Esta expansión del mercado a nivel internacional es una de las características más importantes del desarrollo económico de las empresas en todo el mundo, en la que el uso de la Internet constituye uno de los canales más importante para lograr este objetivo. Esto implica un aumento considerable del número de posibles clientes; estadísticamente hay más posibilidades de que un cliente haga una compra si el contenido se muestra en su lengua materna y el mismo tenga la impresión de que el producto ha sido desarrollado exclusivamente para su país, determinado esencialmente porque muestra un patrón de comportamiento que es compatible con sus expectativas culturales. Por tanto, el desarrollo económico exponencial que espera una empresa implica la adaptación de sus productos o servicios a diferentes mercados, y por tanto tiene el reto de lograr que los mismos tengan una adecuada transformación lingüística, cultural y legal de forma que pueda ser adaptada a diferentes países (de la Torre \& Moxon, 2001) (Norris, 2001) (Oracle, 2005) (Chroust, 2007) (Ishida \& Boeing, 2010) (Esselink, 2000). 
El propósito de este trabajo es reflejar todos aquellos aspectos relacionados con los procesos de Internacionalización y Localización del software, a partir de un estudio actual acerca de estos temas que son muy necesarios para el desarrollo de productos y la prestación de servicios globalizados.

\section{Desarrollo}

Conceptos usados para describir el proceso de transformación de los productos de software o servicios informáticos que se acoplen a otros mercados, como son la Globalización $\left(\mathrm{G} 11 \mathrm{n}^{1}\right)$, Internacionalización (i18 $\left.\mathrm{n}^{2}\right)$ y Localización $\left(110 \mathrm{n}^{3}\right)$ en ocasiones pueden confundirse y por tanto es conveniente realizar una breve explicación de los mismos de forma que se puedan entender claramente y conocer las relaciones que existen entre cada uno de ellos.

\section{Globalización del Software (G11n)}

La globalización de manera general es un fenómeno mundial complejo e irreversible, tuvo sus orígenes en la década de los 80 a partir de cambios radicales de la economía mundial, que hicieron que pequeñas y medianas compañías compitieran fuera de sus mercados tradicionales. Este incremento vertiginoso de la competencia conllevó a crear un amplio estudio de mercado con el propósito de conocer las verdaderas necesidades de sus clientes, por lo que esto constituyó un elemento esencial en el posicionamiento de los mercados siempre competitivos.

A partir de esta expansión global en los mercados, surge la industria de la globalización ${ }^{4}$ la que tuvo precisamente a la industria del software como una de sus principales raíces. El producto software, a diferencia de la mayoría de otros productos, por su portabilidad fue llevado alrededor de todo el mundo con facilidad y a costos relativamente bajos, sin embargo los mismos estaban cargados de contenidos textuales por lo que debieron ser adaptados a lenguajes propios de otras regiones, lo cual conllevó a invertir grandes cantidades de recursos financieros por parte de las empresas. Este proceso de adaptación posibilitó a los desarrolladores de software obtener incrementos en sus ventas al lograr entrar a amplios mercados, a partir de realizar la traducción de textos y de ejecutar tareas técnicas asociadas al rediseño de las interfaces de usuarios.

(Localization Industry Standards Association (LISA), 2007) (Localization Industry Standards Association (LISA), 2003)

Según LISA (Localization Industry Standards Association (LISA), 2007), la globalización es la transformación de negocios y procesos que soportan clientes alrededor de

\footnotetext{
${ }^{1}$ Siglas por las que en ocasiones se reconoce el concepto. El número 11 significa que existen 11 letras entre la 'G' y la 'n' (Ishida \& Boeing, 2010)

${ }^{2}$ Siglas por las que en ocasiones se reconoce el concepto. El número 18 significa que existen 18 letras entre la 'I' y la 'n' (Ishida \& Boeing, 2010)

${ }^{3}$ Siglas por las que en ocasiones se reconoce el concepto. El número 10 significa que existen 10 letras entre la 'L' y la 'n' (Ishida \& Boeing, 2010)

${ }^{4}$ Conocida inicialmente como industria de la localización, como parte de los grandes esfuerzos realizados por las empresas para lograrla globalización de sus productos
} 
todo el mundo, en cualquier idioma, país o cultura que se requiera. A partir de esta definición y ubicando la misma en el contexto del software se reconoce entonces como todas aquellas decisiones organizativas y estratégicas tomadas por una empresa de desarrollo de software para entrar a mercados foráneos a partir de crear las condiciones necesarias para garantizar la adaptación de sus productos o servicios.

Para alcanzar tales propósitos la globalización del software depende de dos elementos claves, la internacionalización (i18n) y la localización (110n), los cuales se muestran como procesos necesarios, inseparables y que se complementan entre sí como se puede mostrar en la figura 1.

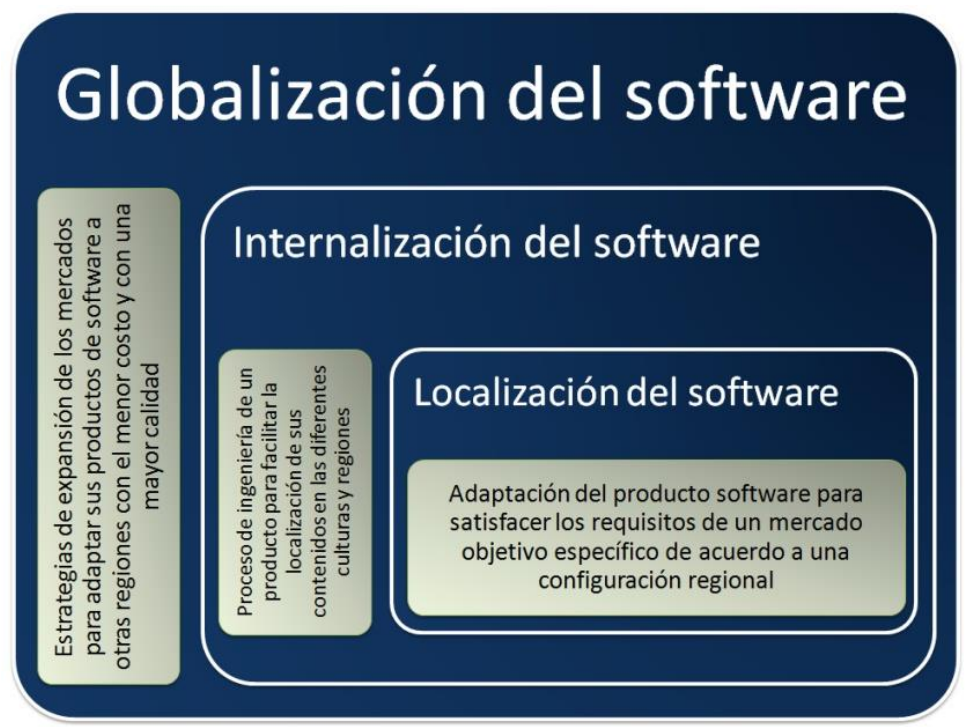

Figura 1. Procesos claves de la globalización del software

LISA (Localization Industry Standards Association (LISA), 2007) presenta estos dos procesos claves de la globalización, como partes que se complementan entre sí dentro del ciclo de desarrollo de un producto de software (Fig. 2). Por ello es preciso que tanto la internacionalización como la localización, requieran de una apropiada planificación dentro del proceso de desarrollo de software convencional para que sea posible la producción de un producto global. Esto conlleva a que las instituciones que producen software deban convertir su proceso de desarrollo convencional en un proceso de desarrollo internacionalizado, donde se incluyen además las etapas de soporte y mantenimiento de acuerdo a las necesidades del mercado. 


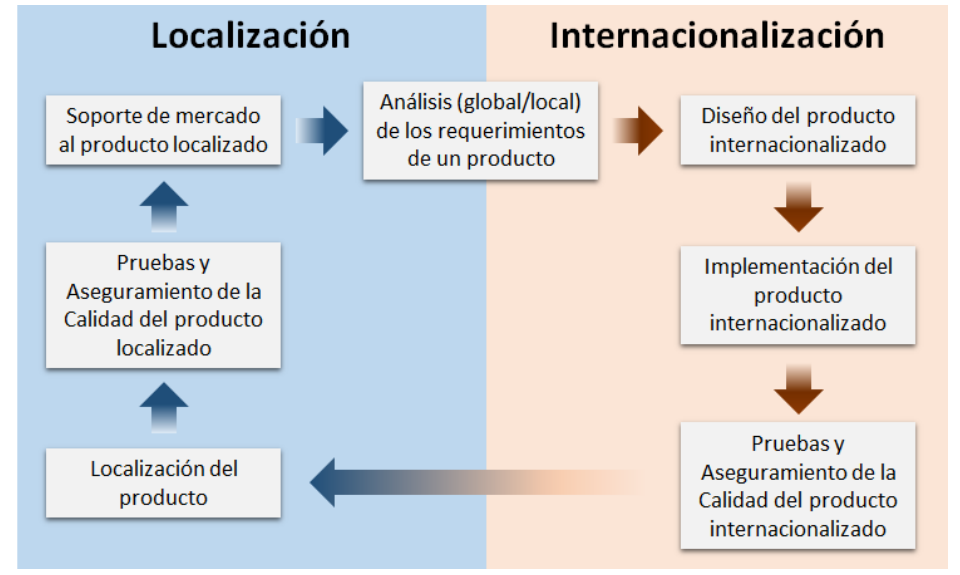

Figura 2. Ciclo de desarrollo de un producto de software global (Localization Industry Standards Association (LISA), 2007) (Localization Industry Standards Association (LISA), 2003)

Como se aprecia en la figura 2, este ciclo comienza con un estudio local y global de los requerimientos del producto deseado, en los que no sólo se incluyen las funcionalidades propias de los negocios sino que se tiene en cuenta un conjunto de especificaciones relacionadas con todos aquellos mercados destinos, este ciclo continua con otro conjunto de pasos propios del proceso desarrollo de un producto internacionalizado y posteriormente pasa a la realización de los pasos esenciales para garantizar el proceso de localización. Lo más importante de este ciclo propuesto consiste precisamente que en toda la institución debe desarrollarse un pensamiento global desde sus primeras fases del proceso de desarrollo con el objetivo de asegurar estos mercados garantizando la calidad del producto y la disminución de sus costos. (Localization Industry Standards Association (LISA), 2007) (Localization Industry Standards Association (LISA), 2003)

En la medida que se desarrolle la presente investigación se verán cada uno de los elementos claves a tener en cuenta para la internacionalización y localización del producto software, alineado al proceso de desarrollo de software.

\section{Internacionalización del Software (i18n)}

Como se ha visto con anterioridad, mientras que la globalización es definida en otras palabra como "el conjunto de decisiones empresariales necesarias desde el punto de vista tecnológico, financiero, gerencial, personal y de mercado para facilitar la adaptación de los productos", la internacionalización (i18n) se define como el proceso de preparar un producto a un nivel técnico, para que pueda ser factiblemente adaptado a múltiples idiomas y convenciones culturales sin la necesidad de ser rediseñado.

En el caso de la industria del software, este proceso no es diferente a lo que se ha definido hasta el momento, y se puede considerar además como un proceso lo suficientemente complejo para dedicarle toda la atención necesaria por parte de las instituciones. La internacionalización se muestra como proceso pero también como la sucesión de un conjunto de pasos que se integran para lograr el desarrollo de un producto global, que es diseñado, desarrollado y garantizada su calidad para el mercado foráneo. Este proceso significa asegurar desde el más alto nivel técnico, 
las condiciones para lograr un proceso factible de adaptación del producto. (Localization Industry Standards Association (LISA), 2007)

La internacionalización del software intenta abstraer las funcionalidades de un producto de cualquier particularidad asociada a la cultura, al idioma o al mercado, de modo que se pueda lograr una adaptación y soporte a los mercados. Si un producto de software no se internacionaliza con antelación, es casi seguro que durante el proceso de localización se incurrirá en un gasto adicional, el cual en algunas ocasiones hará que no sea factible este proceso. Como regla general, se debe asumir que un producto si no está internacionalizado adecuadamente necesitará el doble de tiempo y de costo para localizarlo (Localization Industry Standards Association (LISA), 2007) (Localization Industry Standards Association (LISA), 2003).

El grado de internacionalización requerido depende, en parte, de las complejidades que poseen los lenguajes que se localizan en el producto. Si un producto es desarrollado en un lenguaje base, dígase el Inglés y será localizado al Francés, Italiano, Alemán y Español (las llamadas "FIGS"5, idiomas que tradicionalmente eran las opciones más populares para la localización), el nivel de complejidad de hacer ingeniería de la internacionalización es menor de lo que se requiere si el producto debe ser compatible al Japonés, Chino, Tailandés, Árabe o el Hindi, todo lo cual requiere una planificación y consideraciones de diseño muy especiales (Localization Industry Standards Association (LISA), 2007) (Localization Industry Standards Association (LISA), 2003).

\section{Localización del software (110n)}

Por otro lado, la localización (110n) es el proceso de modificación del producto para reflejar las diferencias en los distintos mercados, ello significa adaptar el producto de manera que pueda ser usado fácilmente por los usuarios finales. En ocasiones se reconoce a este proceso como una "traducción de alta tecnología", sin embargo esta visión no refleja la complejidad y la verdadera importancia de lo que sucede durante la localización de un producto (Localization Industry Standards Association (LISA), 2007). Lograr dar la sensación al usuario que el producto fue diseñado de manera excepcional para su país o región, no es algo que se logre fácilmente, no sólo conlleva a traducir un idioma o a considerar la configuración regional (formato de fecha, medidas, hora y moneda), también conlleva a revisar cómo el producto debe comunicar lo que se quiere, por tanto debe evaluarse el contexto cultural, las creencias, valores y formas de actuación de los diferentes grupos a los cuales va dirigido el producto, entre otros aspectos de gran importancia.

Según LISA (Localization Industry Standards Association (LISA), 2007), en la localización del software comúnmente se tratan los siguientes temas:

Cuestiones lingüisticas: cualquier producto de software que se vende a las personas que no hablan el idioma en el que fue creado requerirá adaptación lingüística. Por ejemplo, una aplicación requerirá la traducción de sus componentes textuales de la interfaz de usuario, la ayuda en línea, la documentación del usuario, los instaladores, entre otros. Más allá del producto en sí, las necesidades de negocio requerirán traducción de materiales colaterales propios del

\footnotetext{
${ }^{5}$ Iniciales de los nombres de los idiomas en inglés: French, Italian, German and Spanish (FIGS)
} 
marketing, como son las páginas web, los materiales de apoyo, y tal vez los documentos de formación, boletines de servicios internos y otros componentes similares (Ishida \& Boeing, 2010).

Cuestiones físicas: se refieren a que la localización de un producto de software a menudo implica la modificación física de los mismos, con el fin de ser aceptado en el mercado local. Estos cambios pueden representar mucho tiempo y costo, y aunque no son muy comunes sí se presentan en software embebidos dentro de equipos de hardware.

Cuestiones de negocios y culturales: refiere que las cuestiones culturales locales pueden afectar los aspectos de diseño de los productos y su localización, ya que deben tener en cuenta las monedas locales empleadas, las convenciones contables, los formatos de las direcciones y teléfonos, los colores y gráficos, e incluso el formato de los nombres debe ser el apropiado para el mercado objetivo. Este tipo de cuestiones a menudo no son contempladas por los diseñadores de productos, simplemente porque no son conscientes de ellos. Sin embargo, a menudo hacen la diferencia entre un producto que funciona a la perfección y tiene éxito en un mercado, en comparación con uno que es frustrante y rechazado por los clientes. Los diseñadores de productos tienen que ser conscientes además de los aspectos políticos y económicos y las expectativas culturales locales. Por ejemplo, las soluciones de comercio electrónico deben tener en cuenta las preferencias de pago locales y métodos, es decir, no pueden asumir que las tarjetas de crédito estarán disponibles en todas partes.

Cuestiones técnicas: considera que dar un correcto soporte a los idiomas locales puede requerir una atención especial en la fase de ingeniería. Por ejemplo, el soporte para idiomas de Asia Oriental que demandan miles de caracteres necesita un diseño y una atención especial, y otros idiomas como el árabe y el hebreo, que se escriben de derecha a izquierda, requieren la adaptación de interfaces de usuario y el uso de las rutinas de manipulación de texto especiales en el software (Ishida \& Boeing, 2010).

De manera general, la decisión de localizar o no un producto de software, hacerlo completamente o de forma parcial dependerá en gran medida de identificar y analizar los riesgos asociados y de los factores que influyen en el grado de localización, entre los que se incluye la naturaleza y alcance del producto, el tamaño del mercado objetivo y la audiencia, la duración del ciclo de vida del producto y las frecuencias de actualización previstas, el comportamiento de los competidores, la aceptación del mercado, y de la legislación nacional o internacional vigente (de la Torre \& Moxon, 2001) (Localization Industry Standards Association (LISA), 2007).

Muchos son los elementos que deben tenerse en cuenta desde los primeros momentos en que se decide estratégicamente la introducción de un producto en diferentes mercados. La importancia de conocerlos impacta de manera significativa en la calidad con que se realice tanto el proceso de internacionalización como el proceso de localización.

\section{Elementos Claves: Internacionalización del Software}

Como se ha expresado con anterioridad, un aspecto esencial del proceso de globalización de un producto de software es precisamente la internacionalización de su proceso de desarrollo, 
lo que significa que el equipo de trabajo debe integrar dentro de este proceso todos aquellos elementos claves asociados a la internacionalización, creando a su vez en cada miembro un pensamiento y una cultura global. Por tanto, siempre debe verse la internacionalización como parte inseparable de las principales actividades del proceso de desarrollo, como son la especificación de requisitos, el diseño, la implementación y las pruebas.

\section{Requerimientos de Software}

Los requerimientos de software cumplen un papel primordial en el proceso de desarrollo de un producto de software, está basado en función de las necesidades planteadas por los clientes en un nivel muy general, donde se descubre, documenta, analiza y se define los servicios o componentes de lo que se desea producir, además de las restricciones que tendrá el producto de software. Su principal tarea consiste en la definición del proceso a seguir en la construcción de un software, y de facilitar la comprensión de lo que el cliente requiera (Sommerville, 2005).

Si bien en el proceso de desarrollo tradicional este paso tiene una gran importancia para el futuro del producto que se desarrolla, en el contexto de un proceso de desarrollo alineado con la internacionalización es el pilar fundamental en el cumplimiento de los objetivos estratégicos de globalización de un producto. Cuando se trata de un mercado nuevo deben estudiarse a profundidad todos los aspectos necesarios asociados a la cultura que se impone en este mercado destino, dentro de los cuales encontramos:

\section{Legislaciones vigentes, las más comunes están asociadas:}

a) Al proceso de negocio que es objeto de informatización.

b) A las políticas de seguridad de la informatización.

c) A las políticas que regulan el uso de determinados dispositivos tecnológicos.

d) A los procesos comerciales y de licencias de uso.

e) A los derechos y protección de la información personal.

f) A los valores éticos y morales de la sociedad.

g) A estándares empleados en las organizaciones (ISO, ITIL, COBIT, entre otras).

\section{Configuraciones regionales:}

a) Formato de fecha, hora, numérico (separadores decimales, de miles, representación de los negativos) y en particular de la moneda (símbolo de la moneda u su ubicación dentro de la representación, o sea si va delante o detrás de la cifra).

b) Formatos asociados con los números de teléfono, direcciones, códigos postales, códigos asociados a diversos elementos del negocio, entre otros.

c) Unidades de medida empleadas.

d) El calendario empleado, en el que el primer día de la semana varía de acuerdo a cada cultura.

\section{Complejidad del lenguaje que se traduce, en el que cada uno de ellos introducen elementos particulares que son considerados de menor o mayor dificultad para el proceso de internacionalización:}


a) Alfabeto (letras, números, símbolos) que se emplea.

b) Conjunto de caracteres necesarios para representar el alfabeto.

c) Estándar usado para representar el alfabeto (ASCII, DBCS, Unicode).

d) Criterios para el ordenamiento alfabético de las palabras (nombres de personas, entidades, entre otros).

e) Dirección en que estos lenguajes manejan su contenido debido a que existen idiomas que se escriben en sentido contrario al que usualmente un desarrollador de software está acostumbrado.

f) Empleo de los diferentes dispositivos de entrada como lo es el teclado.\}

\section{Representación de los contenidos (Interfaz de Usuario):}

a) Elementos de la interfaz de usuario (cajas de texto, listas desplegables, botones, entre otros) que son usados en menor o mayor medida y por tanto deben tenerse en cuenta las preferencias de los usuarios.

b) Disposición de los elementos propios de una interfaz de usuario dentro de las áreas que se representan, existen culturas en las que es muy común que las imágenes estén a la izquierda del texto sin embargo en otras es lo contrario.

c) Los colores son necesarios definirlos de acuerdo a cada cultura por tener diferentes significados.

d) Los elementos visuales como los gráficos, en las diferentes culturas un determinado símbolo pudiera tener un significado totalmente diferente al que se pretende que debiera tener, por lo que debe hacerse mucho énfasis en el uso de aquellos que son más comúnmente usado en cada cultura.

e) Empleo de combinaciones de teclas para acceder a las diferentes funcionalidades del producto que se desarrolla.

\section{Requisitos funcionales propios del negocio:}

Es usual que los productos de software cambien sus requisitos de una cultura a otra, porque cambian los procesos y las reglas de negocio, e incluso bajo una misma pueden existir variaciones. El estudio de estos elementos no comunes asociados a las diferentes culturas a las que debe adaptarse el producto deben ser muy bien identificados de manera que las etapas posteriores al desarrollo los tengan en cuenta.

\section{Restricciones de tecnologías:}

Debe tenerse en cuenta que existen culturas donde resulta poco usual el empleo de algún tipo de tecnologías, por ejemplo las tarjetas de pago, dispositivos de escaneo para la seguridad y protección, entre otros.

Son numerosos los aspectos a tener en cuenta, y por ello el equipo de desarrollo debe estar preparado para asumir una tarea de importancia que define el éxito o no del producto que se desarrolla. 


\section{Diseño de Software}

El diseño de software consiste en tomar decisiones acerca de la organización lógica del producto software que se desarrolla (Sommerville, 2005). Es el proceso mediante el cual se definen la arquitectura, los componentes, interfaces y otras características de un sistema o componente de software (SWEBOK, 2014). El mismo abarca el conjunto de principios, conceptos y prácticas que conducen al desarrollo de un sistema o producto de alta calidad. Los principios de diseño establecen una filosofía primordial que le guiará en el trabajo de diseño que se debe realizar. (Pressman, 2010)

Si bien el levantamiento de requisitos tiene un papel transcendental dentro del ciclo de desarrollo de software, el diseño es una etapa decisiva en el proceso y en particular en la internacionalización del software. En esta etapa como bien se ha planteado, es donde se define la arquitectura que sustentará al producto software que se desea (SWEBOK, 2014), por tanto deben tenerse en cuenta un conjunto de elementos propios de esta etapa de diseño haciendo énfasis en la arquitectura base del software, como son:

1. Lograr el desacoplamiento de las diferentes capas que conforman la estructura de la arquitectura, para la internacionalización del software es muy importante que la arquitectura logre separar la capa de presentación de la capa de negocio, ya que logra que toda la complejidad propia del proceso de localización asociada a la representación de la información se realice de forma independiente.

2. Considerar el uso del estándar de codificación Unicode en todas las capas de la arquitectura y todos los componentes empleados. Unicode, especifica un nombre e identificador numérico único para cada carácter o símbolo, además de otras informaciones necesarias para su uso correcto: direccionalidad, mayúsculas y otros atributos. El estándar trata los caracteres alfabéticos, ideográficos y símbolos de forma equivalente, lo que significa que se pueden mezclar en un mismo texto sin la introducción de marcas o caracteres de control. De manera general, proporciona un número único para cada carácter, sin importar la plataforma, el programa, ni el idioma, permitiendo una fácil transmisión entre distintos sistemas de codificación y plataformas.

3. Maximizar la adaptabilidad de la arquitectura mediante la incorporación de motores de reglas de negocio y de flujos de trabajos, así como la adaptabilidad de su modelo de objetos. La necesidad de adaptarse a los requerimientos de un nuevo mercado conlleva a que se tengan que adaptar las informaciones que se manejan, los documentos, reglas de negocio y flujos de trabajos reflejados como base en el producto software, por tanto se precisa que el producto se adapte a estos requerimientos sin necesidad de ser nuevamente modificado su código fuente.

4. Externalizar la configuración regional y los diferentes idiomas empleados, es vital para la arquitectura puesto que logra que la etapa de localización se realice en el menor tiempo y con el menor costo posible, sin necesidad de modificar el código fuente de la solución de software. Esto significa que se debe crear una capa transversal e independiente relacionada a la internacionalización que agrupe tanto los elementos de configuración regional, como los lenguajes asociados a las culturas propias del mercado destino, de forma que esta capa pueda ser preparada para cada región y sustituida al momento de la localización sin afectar al resto de los componentes que integran la arquitectura. 
5. Crear interfaces de usuarios capaces de adaptarse a los diferentes lenguajes, considerando las especificaciones propias de los lenguajes como son la dirección del texto y de las imágenes que se representan, las longitudes de las cadenas de caracteres que varían de un lenguaje a otro y el espacio destinado para su representación, evitar el uso de texto dentro de imágenes que impidan ser reemplazadas con facilidad en su traducción, entre otros.

6. Cuando se definen los elementos de una base de datos que soporte la internacionalización se deben tener en cuenta las capacidades de internacionalización y localización que proveen cada uno de los Sistemas de Gestión de Bases de Datos a emplear, puesto que estos deben integrarse a la solución que propone la arquitectura. Dentro de estas capacidades se encuentra por ejemplo la de asumir diferentes estándares de codificación y en particular el estándar Unicode para el tratamiento con los caracteres, capacidad para almacenar datos en diferentes formatos, entre otros. En el entorno para el cual se desarrolla existen contenidos que deben ser almacenados en un formato neutro y posteriormente la lógica de la aplicación debe representarla en el formato adecuado, por ejemplo los números, las monedas, entre otros.

7. Considerar un correcto diseño de los esquemas de bases de datos, apegado a los diferentes tipos de informaciones relacionadas con la cultura propia del mercado destino. Durante el diseño de la base de datos, hay valores de contenidos que deben ser almacenados con dependencia de la cultura y por tanto el diseño debe contemplarlos.

8. Seleccionar los COTS (del inglés Commercial Off-The-Shelf) internacionalizados para que se integren a una arquitectura de manera que puedan adaptarse a los diferentes mercados.

9. Determinar la necesidad de internacionalización de estándares para la especificación de la información como lo es el XML ${ }^{6}$, YAML ${ }^{7}$, entre otros.

\section{Implementación del Software}

En esta etapa del desarrollo de software se producen los artefactos más importantes del producto de software, ejemplo de ello es el código fuente (SWEBOK, 2014). Es en esta etapa donde todas las consideraciones propias del diseño son incorporadas al producto. Por tanto deben tenerse en cuenta todas aquellas especificaciones previstas y además otras como lo son:

1. Adecuado uso de un lenguaje controlado en la documentación asociada al producto como lo son la ayuda en línea, el manual de usuario, el manual de instalación, entre otros.

2. Llevar a cabo la internacionalización de estándares como el XML, a partir de las buenas prácticas especificadas por la $\mathrm{W} 3 \mathrm{C}^{8}$.

\section{Pruebas del Software}

El proceso de pruebas de software consiste en la verificación dinámica del comportamiento del producto desarrollado en un conjunto finito de casos de prueba

\footnotetext{
${ }^{6}$ del inglés: Extensible Markup Language (XML)

${ }^{7}$ del inglés: YAML Ain't Another Markup Language

8 del inglés: World Wide Web Consortium, comunidad internacional que desarrolla estándares que aseguran el crecimiento de la Web a largo plazo.
} 
adecuadamente seleccionadas entre el dominio ejecuciones generalmente infinita, contra el comportamiento esperado (SWEBOK, 2014).

En el caso de la etapa de pruebas del software internacionalizado, no se diferencia de la convencional sólo tiene un mayor alcance al existir la necesidad de evaluar todos aquellos elementos asociados a la internacionalización y que fueron especificados en etapas anteriores. En esta etapa es preciso dedicar esfuerzos considerables por ser la etapa donde se evalúa el resultado de todo un proceso de desarrollo y se garantiza la calidad del mismo, lo que significa garantizar el éxito de una estrategia de globalización de una institución y en especial la calidad del producto o servicio que se desea insertar en el mercado internacional.

\section{Elementos Claves: Localización del Software}

Los elementos claves relacionados con el proceso de localización se relacionan específicamente con:

1. Realizar las configuraciones necesarias asociadas al producto y definidas en el paso de especificación del proceso de internacionalización, como son las configuraciones regionales, las traducciones de los contenidos a los diferentes lenguajes, adecuar los flujos de trabajo y de información, entre otros.

2. Un paso importante en la realización de este proceso es la traducción de contenidos visuales y documentos asociados al producto, la cual además de hacerse con la mayor celeridad mediante el empleo de las herramientas, debe contemplar todos los contextos propios de cada lenguaje de manera que el cliente final reciba un mensaje entendible. De lo antes mencionado surge la necesidad de la contratación de personal nativo y profesionales en idiomas para que formen parte de los equipos de localización, logrando así una mejora en el proceso de traducción.

3. Realizar pruebas de localización a partir de las cuales se logren evaluar los resultados de la localización del producto y garantizar con ello la mayor calidad.

\section{Conclusiones}

La internacionalización del software no es un proceso trivial, se necesita tiempo y financiamiento para la realización de profundas investigaciones que conlleven a crear productos de software más competitivos en el mercado internacional. Este proceso fundamental de conjunto con la localización del software no puede verse como un proceso que se realiza una vez desarrollado un producto, sino que deben alinearse al ciclo de desarrollo convencional para lograr desde las primeras etapas incorporar las características asociadas a un producto de software adecuado a las necesidades de los mercados internacionales.

Estos procesos deben ser vistos desde nuestras instituciones de desarrollo de software como parte de lineamientos y pautas claves, desde el momento en que son concebidos como oportunidades de negocio hasta que son desarrollados y ofertados a los clientes, para lograr este objetivo debe crearse una cultura en cada miembro del equipo de trabajo donde todos sean conscientes de las actividades que tienen que hacer en cada etapa. En el caso de nuestras universidades es muy importante incluir estos temas dentro del proceso de formación de 
pregrado y postgrado, puesto que se lograría formar a un profesional más preparado para enfrentar los retos de estos tiempos, de crear mejores productos de software que deben competir en mercados internacionales y lograr como resultado final un posicionamiento clave para nuestra economía.

\section{Bibliografía}

Chroust, G. (31 de 07 de 2007). Software like a courteous butler - Issues of Localization under Cultural Diversity. Proceedings of the 51st Annual Meeting of the ISSS.de la Torre, J., \& Moxon, R. W. (22 de December de 2001). The impact of the information and communication technology revolution on the conduct of international business. 32(4), 617639.

Esselink, B. (2000). A Practical Guide to Localization. Amsterdam \& Philadelphia: John Benjamins.

Hau, E., \& Aparício, M. (2008). Software Internationalization and Localization in Web Based ERP. Retrieved 02 10, 2014, from http://dl.acm.org/citation.cfm?id=1456570

Ishida, R., \& Boeing, K. M. (2010, September 27). Localization vs. Internationalization. (W3C Web Site) Retrieved Enero 10, 2014, from W3C - Internationalization: http://www.w3.org/International/questions/qa-i18n

Localization Industry Standards Association (LISA). (2003). The Localization Industry Primer. Obtenido de http://www.lisa.org

Localization Industry Standards Association (LISA). (2004). Best Practice Guide: Quality Assurance-The Client Perspective. Obtenido de http://www.lisa.org

Localization Industry Standards Association (LISA). (2007). The Globalization Industry Primer: An introduction to preparing your business and products for success in international markets. Recuperado el 01 de febrero de 2014, de http://www.lisa.org

Norris, P. (2001). Digital Dvide: Civic Engagement, Information Poverty and the Internet World Wide. Cambridge University Press.

Oracle. (2005). Oracle Database 10g: The Power of Globalization Technology. Recuperado el 30 de 01 de 2014, de Globalization Support: http://www.oracle.com/technetwork/database/database-technologies/globalization/bwppower-of-globalization-technolo-131243.pdf

Pressman, R. (2010). Software Engineering: A Practitioner's Approach, Seven Edition. Sommerville, I. (2005). Ingeniería del Software (8va Edición).

Swebok. (2014). Guide to the Software Engineering Body of Knowledge. IEEE Computer Society 